\title{
DIFFERENCES OF VECTOR-VALUED FUNCTIONS ON TOPOLOGICAL GROUPS
}

\author{
BOLIS BASIT AND A. J. PRYDE
}

(Communicated by Palle E. T. Jorgensen)

\begin{abstract}
Let $G$ be a locally compact group equipped with right Haar measure. The right differences $\triangle_{h} \varphi$ of functions $\varphi$ on $G$ are defined by $\triangle_{h} \varphi(t)=$ $\varphi(t h)-\varphi(t)$ for $h, t \in G$. Let $\varphi \in L^{\infty}(G)$ and suppose $\triangle_{h} \varphi \in L^{p}(G)$ for some $1 \leq p<\infty$ and all $h \in G$. We prove that $\left\|\triangle_{h} \varphi\right\|_{p}$ is a right uniformly continuous function of $h$. If $G$ is abelian and the Beurling spectrum $s p(\varphi)$ does not contain the unit of the dual group $\hat{G}$, then we show $\varphi \in L^{p}(G)$. These results have analogues for functions $\varphi: G \rightarrow X$, where $X$ is a separable or reflexive Banach space. Finally, we apply our methods to vector-valued right uniformly continuous differences and to absolutely continuous elements of left Banach $G$-modules.
\end{abstract}

\section{$\S 1$. INTRODUCTION}

Let $\xi \in L^{p}(\mathbb{R})$ for some $1 \leq p \leq \infty$. Consider the indefinite integral $\varphi(t)=$ $P \xi(t)=\int_{0}^{t} \xi(x) d x$. Now $\triangle_{h} \varphi(t)=\int_{t}^{t+h} \xi(x) d x=\chi_{h} * \varphi(t)$ where $\chi_{h}$ is the characteristic function of $[-h, 0]$. It follows that $\triangle_{h} \varphi \in L^{p}(\mathbb{R})$ and moreover that $\varphi$ is continuous. We seek conditions under which there exists a constant function $c$ such that $\varphi+c \in L^{p}(\mathbb{R})$. In short we write $\varphi \in L^{p}(\mathbb{R})+\mathbb{C}$.

More generally, let $\varphi \in L^{\infty}(G)$ where $G$ is a locally compact group equipped with right Haar measure and suppose $\triangle_{h} \varphi \in L^{p}(G)$ for some $1 \leq p<\infty$ and all $h \in G$. What additional conditions ensure $\varphi \in L^{p}(G)$ ?

To answer this question, we study the function $\psi(h)=\triangle_{h} \varphi$ and develop a new method for investigating difference problems.

Firstly, let $X$ be a Banach space. The right and left differences of a function $\varphi: G \rightarrow X$ are defined by $\Delta_{h} \varphi(t)=\varphi(t h)-\varphi(t)$ and $\Delta^{h} \varphi(t)=\varphi(h t)-\varphi(t)$ respectively. Let $e$ be the unit in $G$. We say that $\varphi$ is right uniformly continuous if $\lim _{v \rightarrow e} \sup _{t \in G}\left\|\Delta_{v} \varphi(t)\right\|=0$, and let $C_{r u b}(G, X)$ be the space of all right uniformly continuous bounded functions $\varphi: G \rightarrow X$. For functions $f, g: G \rightarrow \mathbb{C}$ we will use the involution given by $f^{*}(t)=f\left(t^{-1}\right)$ and the right convolution $f * g(t)=$ $\int_{G} f\left(t h^{-1}\right) g(h) d h$. The space of compactly supported continuous functions $\varphi: G \rightarrow$ $X$ will be denoted by $C_{c}(G, X)$ or $C_{c}(G)$ if $X=\mathbb{C}$.

In section 2 we prove that the function $\psi$ defined above is right uniformly continuous. This allows us in section 3 to construct a continuous weight function $w$ on

Received by the editors September 21, 1994 and, in revised form, January 4, 1995.

1991 Mathematics Subject Classification. Primary 43A15; Secondary 28B05, 39A05.

Key words and phrases. Differences, weight functions, spectrum, right uniform continuity, $G$ modules, weak continuity, absolutely continuous elements. 
$G$ which dominates $\psi$. The corresponding Beurling algebra $L_{w}^{1}(G)$ is a Wiener algebra (see $[10$, pages $22,83,142]$ ). Under the assumption that $G$ is abelian and the spectrum $s p(\varphi)$ does not contain the unit $\hat{e}$ of the dual group $\widehat{G}$, we use a BochnerHaar integral (see [11, page 132]) to show that $\varphi \in L^{p}(G)$. For the definition of spectrum see (3.1) below ([10, page 139] and [2]). As a consequence, we show that if $\xi \in L^{p}(\mathbb{R})$ for some $1 \leq p \leq \infty$ and if $0 \notin s p(\xi)$, then there exists a constant function $c$ such that $P \xi+c \in L^{p}(\mathbb{R})$. We also show that these results remain valid for $X$-valued functions where $X$ is a separable or reflexive Banach space.

In section 4 we use some of these techniques to prove that vector-valued bounded functions with right uniformly continuous right differences are right uniformly continuous. The abelian case was obtained in [4] and [6]. As a consequence, we obtain in section 5 a characterization of absolutely continuous elements of left Banach $G$-modules.

\section{§2. Technical Lemmas}

Lemma 2.1. Let $\varphi \in L^{\infty}(G)$ and suppose $\triangle_{h} \varphi \in L^{p}(G)$ for some $1 \leq p \leq \infty$ and all $h \in G$. Then the function $\psi: G \rightarrow L^{p}(G), \psi(h)=\triangle_{h} \varphi$, is right uniformly continuous if and only if it is continuous at one point $h_{0} \in G$.

Proof. For arbitrary $h, v \in G$ we have $\|\psi(h v)-\psi(h)\|_{p}=\|\psi(v)-\psi(e)\|_{p}=$ $\left\|\psi\left(h_{0} v\right)-\psi\left(h_{0}\right)\right\|_{p}$ and the lemma follows.

Lemma 2.2. Let $\varphi \in L^{\infty}(G)$ and suppose $\triangle_{h} \varphi \in L^{p}(G)$ for some $1<p<\infty$ and all $h \in G$. Let $g \in L^{q}(G)$ where $\frac{1}{p}+\frac{1}{q}=1$. Then the function $\psi_{g}: G \rightarrow \mathbb{C}$, $\psi_{g}(h)=\int_{G} \triangle_{h} \varphi(t) g(t) d t$, is continuous.

Proof. Firstly let $g \in C_{c}(G)$. Then for $h, v \in G$ we have

$$
\psi_{g}^{*}(h)=\int_{G} \varphi(t) \triangle_{h} g(t) d t \quad \text { and } \quad \Delta_{v} \psi_{g}^{*}(h)=\int_{G} \varphi\left(t h^{-1}\right) \Delta_{v} g(t) d t .
$$

Hence $\psi_{g}^{*}$ is right uniformly continuous . In particular, $\psi_{g}$ is continuous.

Secondly, take an arbitrary $g \in L^{q}(G)$. There exists a sequence $\left\{g_{n}\right\}$ in $C_{c}(G)$ converging to $g$ in the $L^{q}$-norm. This implies $\left|\psi_{g_{n}}(h)-\psi_{g}(h)\right| \rightarrow 0$ as $n \rightarrow \infty$ for all $h \in G$. By the Baire category theorem [11, page 12], $\psi_{g}$ is continuous on a set $D$ of the second category. Since $G$ is locally compact, $D \neq \emptyset$. Now we show that continuity of $\psi_{g}$ at one point $h_{0}$ implies its continuity on $G$. Indeed, note that for $h, k \in G$ we have $\triangle_{k} \psi_{g}(h)=\psi_{g}(h k)-\psi_{g}(h)=\int_{G}[\varphi(t h k)-\varphi(t h)] g(t) d t=$ $\left(\triangle_{k} \varphi\right)^{*} * g\left(h^{-1}\right)$. By [7, $\left.20.32(\mathrm{e})\right], \triangle_{k} \psi_{g} \in C_{0}(G)$. From the identity $\triangle^{v} \psi_{g}(h)$ $=\triangle^{v} \psi_{g}\left(h_{0}\right)+\triangle^{v} \triangle_{h_{0}^{-1} h} \psi_{g}\left(h_{0}\right)$, the continuity of $\psi_{g}$ at $h_{0}$ and the continuity of $\Delta_{h_{0}^{-1} h} \psi_{g}$ at $h_{0}$ we conclude that $\psi_{g}$ is continuous.

Theorem 2.3. Let $\varphi \in L^{\infty}(G)$ and suppose $\triangle_{h} \varphi \in L^{p}(G)$ for some $1<p<\infty$ and all $h \in G$. Then $\psi: G \rightarrow L^{p}(G), \psi(h)=\triangle_{h} \varphi$, is right uniformly continuous.

Proof. By Lemma 2.2, $\psi$ is weakly continuous. That is, $\langle\psi(h), g\rangle=\psi_{g}(h)$ is a continuous function of $h$ for all $g \in L^{q}(G)$. By a Theorem of Namioka [9, Theorem 4.1], $\psi$ is continuous on a dense $G_{\delta}$ subset of $G$. By Lemma 2.1, $\psi$ is right uniformly continuous.

We need the following proposition. 
Proposition 2.4. Let $X$ be a Banach space. Let $\varphi: G \rightarrow X$ be bounded on an open subset $U$ of $G$. Suppose $\triangle_{h} \varphi$ is continuous for each $h \in G$. Then $\varphi$ is continuous.

Proof. For $g \in X^{*}$, the dual of $X$, set $\varphi_{g}=g \circ \varphi$. Then $\varphi_{g}$ is bounded on $U$ and the differences $\Delta_{h} \varphi_{g}=g \circ \Delta_{h} \varphi$ are all continuous. By [1, Theorem 2.1] $\varphi_{g}$ is continuous at each $h_{o} \in U$. From the identity $\Delta^{v} \varphi_{g}(h)=\Delta^{v} \Delta_{h_{o}^{-1} h} \varphi_{g}\left(h_{o}\right)+\Delta^{v} \varphi_{g}\left(h_{o}\right)$ we conclude that $\varphi_{g}$ is continuous on $G$. By [9, Theorem 4.1], $\varphi$ is continuous on a dense $G_{\delta}$ subset of $G$. The identity $\Delta^{v} \varphi(h)=\Delta^{v} \Delta_{h_{1}^{-1} h} \varphi\left(h_{1}\right)+\Delta^{v} \varphi\left(h_{1}\right)$ shows that $\varphi$ is continuous on $G$.

Corollary 2.5. Let $\varphi \in L^{\infty}(G)$ and suppose $\triangle_{h} \varphi \in L^{1}(G)$ for all $h \in G$. Then $\psi: G \rightarrow L^{1}(G), \psi(h)=\triangle_{h} \varphi$, is right uniformly continuous.

Proof. Since $\triangle_{h} \varphi \in L^{1}(G) \cap L^{\infty}(G)$, we conclude $\triangle_{h} \varphi \in L^{p}(G)$ for all $1 \leq p \leq \infty$. By Theorem 2.3, $\left\|\triangle_{h} \varphi\right\|_{1+\frac{1}{n}}$ is a continuous function of $h$ for each $n \in \mathbb{N}$, the natural numbers. Moreover, $\lim _{n \rightarrow \infty}\left\|\triangle_{h} \varphi\right\|_{1+\frac{1}{n}}=\left\|\triangle_{h} \varphi\right\|_{1}$. By the Baire category theorem, $\left\|\triangle_{h} \varphi\right\|_{1}$ is a continuous function of $h$ except on a subset of $G$ of the first category. So it is continuous at some $h_{0} \in G$. Hence there exists a neighbourhood $V$ of the unit $e$ in $G$ such that $\left\|\psi\left(h_{0} v\right)\right\|_{1}=\left\|\triangle_{h_{0} v} \varphi\right\|_{1} \leq 1+\left\|\triangle_{h_{0}} \varphi\right\|_{1}$ for all $v \in V$. Consider the differences $\triangle_{k} \psi$ for $k \in G$. We have $\left\|\Delta_{v} \Delta_{k} \psi(h)\right\|_{1}=\left\|\Delta_{v} \Delta_{k} \varphi\right\|_{1} \rightarrow 0$ as $v \rightarrow e$, by [7, Theorem 20.4], since $\triangle_{k} \varphi \in L^{1}(G)$ for each $k \in G$. Hence $\triangle_{k} \psi: G \rightarrow L^{1}(G)$ is continuous. By Proposition 2.4, $\psi$ is continuous, and by Lemma $2.1, \psi$ is right uniformly continuous.

Remark 2.6. Proposition 2.4 also holds true for the more general case of $\sigma$-well $\alpha$-favorable topological groups as defined in [3]. In this case we use [3, Theorem 1] instead of [9, Theorem 4.1].

Remark 2.7. Let $X$ be a Banach space and $1 \leq p \leq \infty$. Then $L^{p}(G, X)$ denotes the Banach space of strongly measurable functions $\varphi: G \rightarrow X$ for which $\|\varphi(.)\|_{X} \in$ $L^{p}(G)$. If $1 \leq p<\infty$, then $C_{c}(G, X)$ is dense in $L^{p}(G, X)$. Moreover, if $1<p<\infty$ and $X$ is separable or reflexive, then the dual of $L^{p}(G, X)$ is $L^{q}\left(G, X^{*}\right)$, where $\frac{1}{p}+\frac{1}{q}=1$. For this, see $[5,8.20 .3$ and 8.20.5]. It follows that the results of this section remain valid with $L^{p}(G)$ replaced by $L^{p}(G, X)$ for $1 \leq p \leq \infty$ whenever $X$ is a separable or reflexive Banach space.

Remark 2.8. If $X$ is a Banach space not containing a subspace isomorphic to $c_{\circ}$ (the Banach space of convergent to zero complex sequences), then $L^{1}(G, X)$ is also a Banach space not containing a subspace isomorphic to $c_{\circ}$ (see [8]). It follows that in the proof of Corollary 2.5 with $X=\mathbb{C}$ we can avoid application of Proposition 2.4 and conclude instead from [1, Theorem 2.1] that $\psi$ is continuous at some point $h_{o}$ and hence is right uniformly continuous. This simplification is not available for Banach spaces $X$ containing subspaces isomorphic to $c_{\circ}$.

\section{$\S 3$. Bounded functions with Differences in $L^{p}(G)$}

In this section $G$ is a locally compact abelian group. Let $\varphi \in L^{p}(G)$ for some $1 \leq p \leq \infty$. Then [7, Corollary 20.14], $f * \varphi \in L^{p}(G)$ for each $f \in L^{1}(G)$. It follows that

$$
I(\varphi)=\left\{f \in L^{1}(G): f * \varphi=0\right\}
$$


is a closed ideal of $L^{1}(G)$. We define

$$
s p(\varphi)=\text { hull } I(\varphi)=\{\gamma \in \widehat{G}: \hat{f}(\gamma)=0 \text { for all } f \in I(\varphi)\}
$$

where $\widehat{G}$ is the dual group of $G$ and $\hat{f}$ is the Fourier transform of $f$. For the following, see [2, Proposition 1.1].

Proposition 3.1. Let $\varphi \in L^{p}(G)$ for some $1 \leq p \leq \infty$. Then

(i) $\operatorname{sp}(\varphi * f) \subset \operatorname{sp}(\varphi) \cap \operatorname{supp}(\hat{f})$ for all $f \in L^{1}(G)$; and

(ii) $s p(\varphi)=\emptyset$ if and only if $\varphi=0$.

Now let $w$ be a weight function on $G$ satisfying the Beurling-Domar condition. See [10, page 132]. Then the Beurling algebra $L_{w}^{1}(G)=\left\{f \in L^{1}(G): w f \in L^{1}(G)\right\}$ is a Wiener algebra $[10,6.3 .1]$. The dual of $L_{w}^{1}(G)$ is $L_{w}^{\infty}(G)=\left\{\varphi: \frac{\varphi}{w} \in L^{\infty}(G)\right\}$. If $\varphi \in L_{w}^{\infty}(G)$, then its spectrum with respect to $L_{w}^{1}(G)$ will be denoted by $\operatorname{sp}_{w}(\varphi)=$ hull $I_{w}(\varphi)$, where $I_{w}(\varphi)=\left\{f \in L_{w}^{1}(G): f * \varphi=0\right\}$ (see [10, page 142]).

We show

Theorem 3.2. Let $\varphi \in L^{\infty}(G)$ and suppose $\triangle_{h} \varphi \in L^{p}(G)$ for some $1 \leq p<\infty$ and all $h \in G$. If $\hat{e} \notin s p(\varphi)$, then $\varphi \in L^{p}(G)$.

Proof. By Theorem 2.3 and Corollary 2.5, $\psi: G \rightarrow L^{p}(G), \psi(h)=\triangle_{h} \varphi$, is uniformly continuous. Define $w: G \rightarrow \mathbb{R}$ by $w(h)=1+\|\psi(h)\|_{p}+\left\|\psi\left(h^{-1}\right)\right\|_{p}$. Then

(i) $w(h k) \leq w(h) w(k)$ for all $h, k \in G$;

(ii) $w$ is uniformly continuous; and

(iii) $w\left(h^{n}\right) \leq n w(h)$ for all $h \in G, n \in \mathbb{N}$.

It follows that $w$ is a weight function on $G$ satisfying the Beurling-Domar condition. Hence $L_{w}^{1}(G)$ is a Wiener algebra. Since $\hat{e} \notin s p(\varphi)$, by $[10,2.1 .3$, Remark] there exist a neighbourhood $V$ of $\hat{e}$ and a function $f \in L_{w}^{1}(G)$ such that $\operatorname{supp}(\hat{f}) \subset V, \hat{f}(\hat{e})=1$ and $V \cap s p(\varphi)=\emptyset$. By Proposition 3.1, $\varphi * f=0$. Hence $\varphi(t)=\int_{G}\left[\varphi(t)-\varphi\left(t s^{-1}\right)\right] f(s) d s=-\int_{G} \triangle_{s^{-1}} \varphi(t) f(s) d s$. The integrand as a function of $s$ from $G$ to $L^{p}(G)$ is weakly Borel measurable. Moreover, we claim that it is almost separably-valued with respect to Haar measure. Indeed $f \in L^{1}(G)$, so its (essential) support is $\sigma$-compact. The function $\triangle_{s^{-1}} \varphi$ of $s$ is continuous and, therefore, restricted to the support of $f$ it has a range which is $\sigma$-compact in $L^{p}(G)$ and hence separable. The claim follows. By Pettis's theorem [11, page 131] the integrand is strongly Borel measurable. Moreover, since $\left\|\triangle_{s^{-1}} \varphi\right\|_{p} \leq w(s)$, by Bochner's theorem [11, page 133] the Bochner-Haar integral $\int_{G} \triangle_{s^{-1}} \varphi f(s) d s$ exists and belongs to $L^{p}(G)$. That is, $\varphi \in L^{p}(G)$.

Letting $w$ denote the weight function defined above, one can show by the same method as used in the proof of Theorem 3.2.

Theorem 3.3. Let $\varphi \in L^{\infty}(G)$ and suppose $1 \leq p<\infty$. Then $\varphi \in L^{p}(G)$ if and only if

(i) $\triangle_{h} \varphi \in L^{p}(G)$ for all $h \in G$, and

(ii) there exists $f \in L_{w}^{1}(G), f \neq 0$, such that $f * \varphi \in L^{p}(G)$.

Similarly, we have 
Theorem 3.4. Let $\varphi \in L^{\infty}(G)$ and suppose $\triangle_{h} \varphi \in L^{p}(G)$ for some $1 \leq p<\infty$ and all $h \in G$. If $f \in L_{w}^{1}(G)$ and $\hat{f}(\hat{e})=1$, then $\varphi-f * \varphi \in L^{p}(G)$.

To study indefinite integrals, we use the weight

$$
v(x)=1+|x|, x \in \mathbb{R} .
$$

It is readily seen that $v$ is a symmetric weight function satisfying Beurling conditions (see [10, page 17]). It follows that $L_{v}^{1}(\mathbb{R})$ is a Wiener algebra. We denote by $C_{u}(\mathbb{R})$ $\left(C_{u b}(\mathbb{R})\right)$ the set of all complex-valued uniformly continuous (uniformly continuous bounded) functions defined on $\mathbb{R}$.

Proposition 3.5. If $\xi \in L^{p}(\mathbb{R})$ where $1 \leq p \leq \infty$ and $v$ is given by (3.2), then $P \xi \in C_{u}(\mathbb{R}) \cap L_{w}^{\infty}(\mathbb{R})$.

Proof. If $p=1$, it is well-known that $P \xi$ is absolutely continuous and hence uniformly continuous. For arbitrary $p$, and $x, h \in \mathbb{R},|P \xi(x+h)-P \xi(x)|=$ $\left|\int_{0}^{h} \xi(x+t) d t\right| \leq|h|^{1-1 / p}\|\xi\|_{p}$ showing that $P \xi \in C_{u}(\mathbb{R})$. Moreover, $|P \xi(x)|=$ $\left|\int_{0}^{x} \xi(t) d t\right| \leq|x|^{1-1 / p}\|\xi\|_{p}$, showing that $P \xi \in L_{w}^{\infty}(\mathbb{R})$.

Theorem 3.6. If $\xi \in L^{p}(\mathbb{R})$ where $1 \leq p \leq \infty$ and $0 \notin s p(\xi)$, then $P \xi \in C_{u b}(\mathbb{R})$. Moreover, $P \xi \in L^{p}(\mathbb{R})+\mathbb{C}$.

Proof. Since $0 \notin s p(\xi)$, there exists a neighbourhood $V=[-\delta, \delta]$ such that $s p(\xi) \cap$ $V=\emptyset$. Since $L_{v}^{1}(\mathbb{R})$ is a Wiener algebra, there is a function $h \in L_{v}^{1}(\mathbb{R})$ such that $\hat{h}=$ 1 for $|\lambda| \leq \delta / 4$ and $\hat{h}=0$ for $|\lambda| \geq \delta / 3$. By Proposition 3.1, $h * \varphi=0$. Similarly, by [10, page 140-141] and [2, Proposition 1.1], $s p_{v}(h * P \xi) \subset \operatorname{supp}(\hat{h}) \cap s p_{v}(P \xi) \subset\{0\}$. Since $\frac{d(h * P \xi)}{d x}=h * \xi=0$, we conclude that $h * P \xi=c$, a constant. If $\eta=P \xi-c$, then $0 \notin s p_{w}(\eta)$. Indeed, $h * \eta=h * P \xi-h * c=c-c=0$. Thus $h \in I_{v}(\eta)$ and $\hat{h}(0)=1$, showing $0 \notin s p_{w}(\eta)$. By Proposition 3.5, $\eta \in C_{u}(\mathbb{R})$ and so by $[2$, Theorem 4.2], $\eta$ is bounded and so is $P \xi$. This proves that $P \xi \in C_{u b}(\mathbb{R})$. The function $\eta$ satisfies all the conditions of Theorem 3.2, therefore $\eta \in L^{p}(\mathbb{R})$. Hence $P \xi \in L^{p}(\mathbb{R})+\mathbb{C}$.

Remark 3.7. The results of section 3 remain true for $X$-valued functions provided that $X$ is separable or reflexive. The spectrum of $\varphi \in L^{p}(G, X)$ is defined again by (3.1).

\section{§4. Right uniformly Continuous Differences}

In this section and the next, we again take a locally compact group $G$ and a Banach space $X$. The following theorem, for the case of abelian groups, was proved by Datry and Muraz [4, Théorème 4, Corollaire]. Their proof was indirect, using deep results for Banach $G$-modules. We give a different direct proof, using the techniques of the previous sections, and then deduce results for $G$-modules in section 5 .

Theorem 4.1. Let $\varphi: G \rightarrow X$ be a bounded function and suppose $\triangle_{h} \varphi \in$ $C_{\text {rub }}(G, X)$ for all $h \in G$. Then $\varphi \in C_{\text {rub }}(G, X)$.

Proof. Define $\psi: G \rightarrow C_{r u b}(G, X)$ by $\psi(h)=\triangle_{h} \varphi$. Then for $h, k \in G$ we have

$$
\left\|\Delta_{v} \Delta_{k} \psi(h)\right\|_{\infty}=\left\|\Delta_{v} \Delta_{k} \varphi\right\|_{\infty} \rightarrow 0 \quad \text { as } v \rightarrow e .
$$


So $\Delta_{k} \psi: G \rightarrow C_{r u b}(G, X)$ is continuous. By Proposition 2.4, $\psi$ is continuous. Finally, continuity of $\psi$ at $e$ implies that $\varphi$ is right uniformly continuous.

Remark 4.2. In view of Remark 2.6, Theorem 4.1 holds true for the more general case of $\sigma$-well $\alpha$-favorable topological groups.

\section{$\S 5$. Application to Left $G$-MOdules}

A Banach space $X$ together with a family of bounded linear operators $A_{h}: X \rightarrow$ $X$, for $h \in G$, is called a left Banach $G$-module if

(i) $A_{e}(x)=x$ for all $x \in X$;

(ii) $A_{h k}(x)=A_{h}\left(A_{k}(x)\right)$ for all $h, k \in G$ and all $x \in X$;

(iii) $\left\|A_{h}(x)\right\| \leq \kappa\|x\|$ for all $h \in G$, all $x \in X$, and some $\kappa>0$.

The space $X_{a b s}=\left\{x \in X:\left\|A_{v} x-x\right\| \rightarrow 0\right.$ as $\left.v \rightarrow e\right\}$ is a closed submodule of $X$. Its elements are called absolutely continuous. See Datry and Muraz [4], where Theorem 5.2 below is obtained in the case $G$ is abelian using a different proof.

For a fixed vector $x \in X$ we study the function $\psi: G \rightarrow X$ given by $\psi(h)=A_{h} x$. So $x$ is absolutely continuous if and only if $\psi$ is continuous at $e$. In fact the following is true.

Theorem 5.1. For an element $x$ of a left Banach $G$-module $X$, the following are equivalent.

(a) $x \in X_{a b s}$;

(b) $\psi$ is weakly continuous at some point in $G$;

(c) $\psi$ is right uniformly continuous.

Proof. If $x \in X_{a b s}$, then $\psi$ is continuous, and therefore weakly continuous, at $e$. So (a) implies (b). Next suppose $\psi$ is weakly continuous at $h_{0}$. From the identity $\left\langle\triangle_{v} \psi(h), x^{*}\right\rangle=\left\langle\triangle_{v} \psi\left(h_{0}\right), A_{h h_{0}^{-1}}^{*}\left(x^{*}\right)\right\rangle$ for $h, v \in G$ and $x^{*} \in X^{*}$, it follows that $\psi$ is weakly continuous on $G$. By $\left[9\right.$, Theorem 4.1] $\psi$ is continuous at some point $h_{1}$. From the identity $\triangle_{v} \psi(h)=A_{h h_{1}^{-1}}\left(\triangle_{v} \psi\left(h_{1}\right)\right)$ it follows that $\psi$ is right uniformly continuous. Hence (b) implies (c). That (c) implies (a) is obvious.

Theorem 5.2. Let $x$ be an element of a Banach $G$-module $X$. If $A_{h} x-x \in X_{a b s}$ for all $h \in G$, then $x \in X_{\text {abs }}$.

Proof. For each $k \in G, \triangle_{k} \psi(h)=A_{h}\left(A_{k} x-x\right)$ which by Theorem 5.1 defines a right uniformly continuous function $\triangle_{k} \psi: G \rightarrow X$. As $\psi$ is bounded, Theorem 4.1 shows that $\psi$ is right uniformly continuous. Hence $x \in X_{a b s}$.

Remark 5.3. In view of Remark 2.6, the results of this section hold true for the more general case of $\sigma$-well $\alpha$-favorable topological groups.

\section{REFERENCES}

1. B. Basit and M. Emam, Differences of functions in locally convex spaces and applications to almost periodic and almost automorphic functions, Annales Polonici Math. XLI (1983), 193-201. MR 85d:43005

2. B. Basit and A.J. Pryde, Polynomials and functions with finite spectra on locally compact abelian groups, Bull. Austral. Math. Soc. 51 (1994), 33-42. CMP 95:07

3. J.P.R Christensen, Joint continuity of separately continuous functions, Proc. Amer. Math. Soc. 82 (1981), 455-461. MR 82h:54012

4. C. Datry and G. Muraz, Analyse harmonique dans les modules de Banach I: propriétés générales, Bull. Science Mathematique 119 (1995), 299-337. 
5. R.E. Edwards, Functional Analysis - Theory and Applications, Holt, Rinehart and Winston Inc., New York, 1965. MR 36:4308

6. F. Galvin, G. Muraz et P. Szeptycki, Fonction aux différence $f(x)-f(a+x)$ continues, C.R.Acad.Sci. Paris, série I 315 (1991), 397-400. MR 94b:39035

7. E. Hewitt and K.A. Ross, Abstract Harmonic Analysis, Part I, Springer-Verlag, 1979. MR 81k:43001

8. S. Kwapien, On Banach spaces containing $c_{o}$, Studia Math. 52 (1974), 187-188. MR 50:8627

9. I. Namioka, Separate continuity and joint continuity, Pacific Journal of Math. 51 (1974), 515-531. MR 51:6693

10. H. Reiter, Classical Harmonic Analysis and Locally Compact Groups, Oxford Math. Monographs, Oxford Univ., 1968. MR 46:5933

11. K. Yosida, Functional Analysis, Springer-Verlag, Berlin, Heidelberg, New York, 1966. MR 50:2851

Department of Mathematics, Monash University, Clayton, Victoria 3168, Australia

E-mail address: bbasit (ajpryde)@vaxc.cc.monash.edu.au 